\title{
ALGORITMO PARA ESTUDOS DE PERCOLAÇÃO EM HIPER-REDES
}

\author{
D. Ecco', D. Teixeira', J. E. de Freitas ${ }^{2}$ e L. R. da Silva ${ }^{3}$ \\ Instituto Federal de Educação, Ciência e Tecnologia do Rio Grande do Norte (IFRN) \\ Universidade Federal do Rio Grande do Norte (UFRN) \\ daniel.ecco@ifrn.edu.br* \\ Artigo submetido em julho/2015 e aceito em outubro/2015 \\ DOI: 10.15628/holos.2015.3245
}

\section{RESUMO}

Através de estudos sobre percolação, pode-se determinar se uma rede bidimensional percola, percorrendo apenas parte das fronteiras dos aglomerados, verificando se existem dois sítios da fronteira em lados opostos da rede, isto é, sem a necessidade de preencher todos os sítios que formam os aglomerados. Isto nos motivou a desenvolver um algoritmo que vamos descrever neste trabalho. Diante da velocidade que este algoritmo terá em percorrer apenas parte das fronteiras dos aglomerados, vimos que seria possível estudar redes de tamanhos jamais alcançados (superiores a um trilhão de sítios), com um baixo custo computacional e complexidade menor que 1 em relação aos algoritmos já desenvolvidos sobre o tema percolação. Passamos, com isso, a querer estudar o comportamento do limiar de percolação em redes dos mais diversos tamanhos e com uma grande quantidade de simulações, as quais os resultados permitiram fazer comparações e confirmar as previsões feitas através de leis de escalas já conhecidas na literatura.

PALAVRAS-CHAVE: Limiar, Percolação, Sequências com Repetição, Fronteira e Aglomerado Percolante.

\section{ALGORITHM FOR PERCOLATION STUDIES IN HIPER - NETWORKS}

\begin{abstract}
Through studies of percolation, we realized that we could determine whether a two-dimensional percolating network, covering only part of the borders of the clusters, making sure that there are two sites of the border on opposite sides of the net, that is, without the need to fill all sites that form pellets. This motivated us to develop an algorithm that we describe in this paper. Faced with the speed that this algorithm will go in only part of the borders of clusters, we saw that it would be possible to
\end{abstract}

study never reached sizes of networks (more than a trillion websites), with a low computational cost and lower complexity 1 for algorithms already developed on the subject percolation. We spent, therefore, to want to study the percolation threshold behavior in networks of different sizes and with a lot of simulations, which allowed the results and make comparisons confirm the predictions made by scales laws already known in the literature.

KEYWORDS: Threshold, Percolation, Sequences with Repetition, Border and Cluster Percolating. 


\section{INTRODUÇÃO}

Muitos sistemas físicos, químicos, biomatemáticos, econômicos, geológicos, na área de exploração petrolífera, entre outros são formados por um grande número de elementos que interagem entre si [1]. Estes sistemas possuem alta complexidade, se comportam de forma não linear e são representados por modelos matemáticos muitas vezes sem solução analítica [2, 3]. Nestes casos são necessárias simulações numéricas para estudar e compreender estes sistemas. Desta forma, desenvolvemos um algoritmo eficiente e ultra veloz, tendo como objetivo tornar possível e viável simular alguns destes problemas.

A teoria da percolação constitui um exemplo de modelo matemático que pode ser utilizado para representar alguns sistemas de alta complexidade [1, 4]. É um ramo da teoria das probabilidades que trata das propriedades dos meios aleatórios, possui uma definição simples em termos geométricos, apesar de não ter solução analítica no caso geral e apresenta características não triviais do ponto de vista da Física, incluindo fenômenos críticos, fractalidade e a não linearidade [5]. Os primeiros processos de percolação foram desenvolvidos por Paul J. Flory (1941) e Walter H. Stockmayer (1943), com o objetivo de descrever como as ramificações de pequenas moléculas reagiam para formar moléculas muito grandes. Entretanto, Flory e Stockmayer não denominaram esta teoria como sendo um processo de percolação [6], sendo aprofundada por Dietrich Stauffer [1].

Alguns fenômenos que podemos citar como estudados pela teoria da percolação são: transição de fase condutor/isolante, propagação de fogo em florestas, epidemias, polímeros ramificados, recuperação de petróleo pela injeção de água ou de um gás miscível e fluxos de fluidos em meios porosos [7]. Reservatórios de petróleo, por exemplo, possuem propriedades de fractalidade, pois apresentam grandes heterogeneidades geológicas em um amplo intervalo de escalas de comprimento, desde centímetros até quilômetros, mantendo similaridades [8]. Testemunhos e poços de testes tem boa representatividade, mas os resultados devem ser interpretados para inferir propriedades de fluxo, quando, por sua vez, o fluxo ocorre na escala dos poros. Portanto, existe uma grande incerteza a ser considerada. Este tipo de abordagem pode ser feito através da simulação computacional das equações de Navier Stokes, em um modelo aproximado que gera resultados imprecisos, com um alto custo computacional. Por isto, existe um grande estímulo à produção de modelos alternativos mais simples que possam predizer as incertezas [9]. Tomando-se como exemplo o fluxo de um fluido num reservatório de petróleo, vemos que o mesmo é afetado por heterogeneidades, tornando-se necessária a construção de modelos matemáticos para se fazer o estudo de determinadas simulações. Um modelo matemático para representar as conectividades e transportes em sistemas heterogêneos e geometricamente complexos é obtido através da teoria da percolação.

Alguns tipos de percolação que podemos citar dentre os modelos discretos são, percolação por sítios, por ligações e por sítios e ligações. O modelo de percolação por sítios em uma rede quadrada é o mais simples, sendo usado, até mesmo, para definir a própria percolação. Tomemos uma rede quadrada, de comprimento linear $L$, e consideremos que cada sítio da rede seja ocupado, aleatoriamente, com probabilidade $p$, ou permaneça vazio com probabilidade 1 - $p$. Dizemos que dois sítios são vizinhos se possuem dois lados em comum. Se dois sítios vizinhos estão ocupados, então dizemos que eles formam um aglomerado de dois sítios ocupados [10,11]. Para pequenos valores de $p$, a figura resultante mostrará a maioria das células ocupadas isoladas, circundadas por 
células não ocupadas. As células ocupadas passarão a formar agrupamentos à medida que a probabilidade $p$ aumenta. Na Figura 1 tem-se três casos de ocupações com $p$ igual a 0,20, 0,40 e 0,60 .

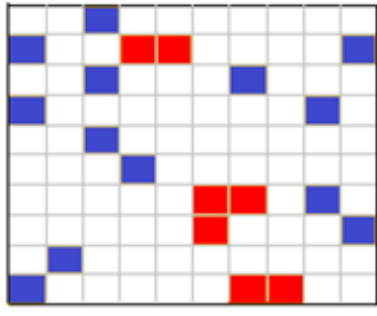

(a)

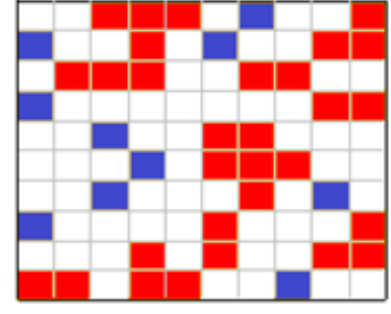

(b)

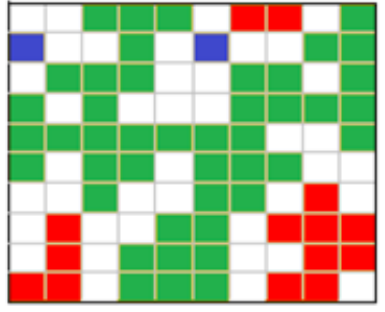

(c)

Figura 1: Redes com 10 x 10 sítios e diferentes probabilidades de ocupação.

Dizemos que a rede percola para um determinado valor da probabilidade de ocupação $p$, denotado por $p_{c}$, ao surgir um aglomerado que, possivelmente torna-se dominante sobre os outros e pode conectar margens opostas da rede, sendo denominado de aglomerado percolante. Denominamos a probabilidade $p_{c}$, para a qual o sistema começa a percolar, de limiar de percolação ou concentração crítica, pois separa fases distintas (isolantes e condutoras). No caso de redes de tamanho linear infinito, o limiar de percolação conhecido na literatura para o caso da rede quadrada é $p_{c}=0,5927$.

O limiar de percolação é a característica fundamental da teoria da percolação. Para valores de $p$ maiores que $p_{c}$, os aglomerados formados (considerados finitos) são absorvidos pelo aglomerado percolante $[2,10]$, conectando margens opostas da rede, como visto na Figura 1 [11]. Para valores de $p$ menores que $p_{c}$ o sistema é isolante, uma vez que não existe nenhum caminho que conecte duas margens opostas da rede.

\section{METODOLOGIA}

Desenvolvemos e executamos o algoritmo no sistema operacional Windows 8, 64bits, processador Intel i7, 4 processadores lógicos de $3 \mathrm{Ghz}$ de velocidade, memória Ram de $8 \mathrm{~Gb}$ e HD de $500 \mathrm{~Gb}+32 \mathrm{~Gb}$ SSD.

\subsection{Dados para as estatísticas}

Sendo $L$ o lado da rede quadrada, fizemos experiências com $L=2^{10}, 2^{11}, \ldots, 2^{23}$. A rede maior possui $2^{23} \times 2^{23}=2^{46}$ sítios. Definimos $p$ como Probabilidade crítica, o qual é informado e será divisor do intervalo aberto $(a, b)$ e candidato a $p_{c}$. O programa é executado $\operatorname{com} p$ variando

dentro de um intervalo aberto $(a, b)$, com pontos equidistantes, onde $\frac{(a+b)}{2}$ é próximo do limiar de percolação 0,5927 já estudado. Para cada $L$, procuramos executar um número suficiente de vezes, para estabilizar as curvas, fractalidade da fronteira do aglomerado percolante e densidade de percolação que fizemos. Para estudos em uma rede de lado $L$, podemos obter informações sobre percolação para qualquer rede menor que $L$.

Os números foram gerados pelo KPZ(KPS). Este, por sua vez, apresentou um vício na sequência de números aleatórios para redes muito grandes, provocando ausência de percolação nestas redes. Para corrigir este vício, geramos 500 distribuições homogêneas paralelas e ordenadas 
pelo KPZ. O primeiro sorteio foi tirado da distribuição de ordem 1. O segundo sorteio foi tirado da distribuição de ordem 2 e assim sucessivamente até a ordem 500. De 501 em diante, sorteia-se da mesma forma. A nova sequência de sucessos apresentou-se sem os vícios para a verificação e formação de estatísticas de percolação.

Cada execução possui uma probabilidade $p$ informada $\left(P_{\text {inf }}\right)$ que deveria coincidir com a frequência relativa de sucessos, obtida pelo gerador de números aleatórios. Como nem sempre coincide, é interessante ter o controle das frequências relativas obtidas, gerando duas estatísticas, uma para o $P_{\text {inf }}$ e outra para $p$ igual a frequência realizada.

\subsection{Sequência sem repetição}

Os extremos dos subintervalos do intervalo máximo $(b-a)$ são termos de uma sequência. Define-se como:

Dados $(b-a)$ e $B$ com $a, b \in \mathbb{R}$ e $B \in I^{+}$, considere a sequência $S=\left\{x_{n}\right\}, n \in I^{+}$com subsequências $S_{L}, L=1,2, \ldots$ com $B^{L}-1$ elementos, que subdividem $(b-a)$ em $B^{L}$ subintervalos de comprimentos $\frac{(b-a)}{B^{L}}$. Vamos completar $S_{L-1}$ para obter $S_{L}$. Para isto, devemos acrescentar $B^{L}-B^{L-1}$ elementos. $S=\left\{x_{n}\right\}$ é uma sequência numérica que divide o intervalo (a, b) em subintervalos de igual comprimento.

Na Tabela 1 temos $B=2, a=32$ e $b=48$. Assim, para $L=1$ obtemos as sequência $S_{1}$ com 1 elemento, para $L=2$ obtemos as sequência $S_{2}$ com 3 elementos, para $L=3$ obtemos as sequência $S_{3}$ com 7 elementos e assim sucessivamente.

Para calcular $x_{n}$, seja $L$ tal que $B^{L-1}-1<n \leq B^{L-1}$. Na sequência $S_{L-1}$ tem $B^{L-1}-1$ elementos. Na sequência $S_{L}$ incluímos mais $B^{L}-B^{L-1}$ elementos com intervalo $\frac{b-a}{B^{L}}$. Então, aplicamos a quantidade de pontos e acrescentamos mais um ponto, de forma que os pares são os novos e os ímpares os anteriores. Então,

$i=\left[n-\left(B^{L-1}-1\right)-1\right] .2$

Por fim,

$x_{n}=a+i \cdot \frac{(b-a)}{B^{L}}$.

Tabela 1: Sequência $x_{n}$ sem repetição.

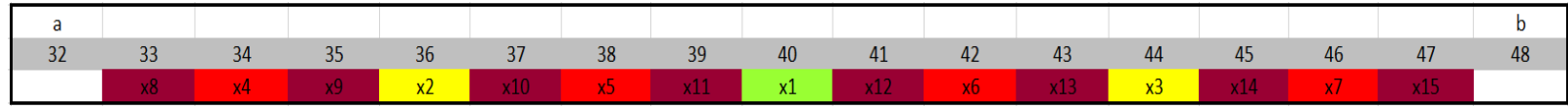

\subsection{Sequência com repetição}

Vamos definir agora a sequência $T=\left\{y_{n}\right\}$. Dados $(b-a)$ e $B$ com $a, b \in \mathbb{R}$ e $B \in I^{+}$, considere a sequência $T=\left\{y_{n}\right\} n \in I^{+}$com subsequências $S_{L}, L=1,2, \ldots$ com $B^{L}-$ 1 elementos, que subdividem $(b-a)$ em $B^{L}$ subintervalos de comprimentos $\frac{(b-a)}{B^{L}}$. Representemos por $B_{l}(L)$ o bloco de ordem $L$, formado pelas rep $(L)$ repetições de linhas com os elementos de $S_{B^{L}-1}$, onde $\operatorname{rep}(L): I^{+} \rightarrow I^{+}$é uma função não decrescente. $T=\left\{y_{n}\right\}$ é uma 
sequência numérica que divide o intervalo $(a, b)$ em subintervalos de igual comprimento, permitindo repetições segundo a regra $\operatorname{rep}(L)$, que permite crescer a pesquisa, aumentando a frequência, usando a pesquisa anterior.

Raciocinemos por indução, tendo formado $B_{l}(L-1)$, como formar $B_{l}(L)$ ? Inicialmente completamos as linhas de $B_{l}(L-1)$ para ficar com $\operatorname{rep}(L-1) \cdot\left(2^{L}-1\right)$ elementos, para isto devemos acrescentar a cada linha $\left(2^{L}-2^{L-1}\right)$ elementos, totalizando um incremento de $\operatorname{rep}(L-1) .\left(2^{L}-2^{L-1}\right)$ elementos. Em seguida, para concluir $B_{l}(L)$ acrescenta-se $\operatorname{rep}(L)-$ $\operatorname{rep}(L-1)$ linhas com $2^{L-1}-1$ elementos, totalizando um novo incremento de (rep $(L)-$ $\operatorname{rep}(L-1)) \cdot\left(2^{L-1}-1\right)$ elementos.

Considerando que cada linha, complementar ou não, é formada da esquerda para direita, como calcular $y_{n}$ ? Temos dois casos:

10 caso:

$\operatorname{rep}(L-1) \cdot\left(B^{L-1}-1\right)<n \leq \operatorname{rep}(L)\left(B^{L-1}-1\right)$

2o caso:

$\operatorname{rep}(L) .\left(B^{L}-1\right)<n \leq \operatorname{rep}(L)\left(B^{L}-1\right)$

10 caso: cada linha do 1 ㅇ complementar tem $B^{L}-B^{L-1}$ elementos, de uma forma que a cada $(B-1)$ elementos salta uma posição, ocupada anteriormente. Então, $J=1,2, \ldots, B^{L}-$ $B^{L-1}$, a posição de ordem de $y_{n}$ na linha complementar, será

$\left.j=1+(n-\operatorname{rep}(L-1)) \cdot\left(B^{L-1}-1\right)-1\right) \%\left(B^{L-1}-1\right)$

20 Caso: o índice $i=1,3, \ldots, B^{L}-1$ é a posição relativa de $y_{n}$ na linha com $B_{l}(L-1)$ elementos e será

$i=\left(\frac{j-1}{B-1}\right)_{i n t} \cdot B+1+(j-1) \%(B-1)$

Destacamos que $\left.\left(B^{L-1}-1\right)-1\right) \%\left(B^{L-1}-1\right)$ é o resto da divisão de $\left.\left(B^{L-1}-1\right)-1\right)$ por $\left(B^{L-1}-1\right)$, assim como $(j-1) \%(B-1)$ é o resto da divisão de $(j-1)$ por $(B-1)$.

Por fim,

$y_{n}=a+i \cdot \frac{(b-a)}{B^{L}}$.

Para cada tamanho de rede, paramos em uma quantidade de execuções específica $B_{l}(L)=$ $\left(2^{L}-1\right)^{2}$, para que todos os $P_{\text {inf }}$ tenham a mesma frequência. $O$ centro do intervalo é variado de acordo com a procura do limiar de percolação, sendo a média de a e b. $O$ divisor, dado em potência de 2, indica em quanto devemos dividir o intervalo máximo para obter o intervalo de pesquisa na execução. Para executarmos o programa, informamos o centro, o intervalo máximo e o divisor. Apenas o comprimento do intervalo máximo permanece constante a $(b-a)$. 
Tabela 2: Sequência $y_{n}$ com repetição, centro 0,592745, intervalo máximo 0,000128 e divisor 16 .

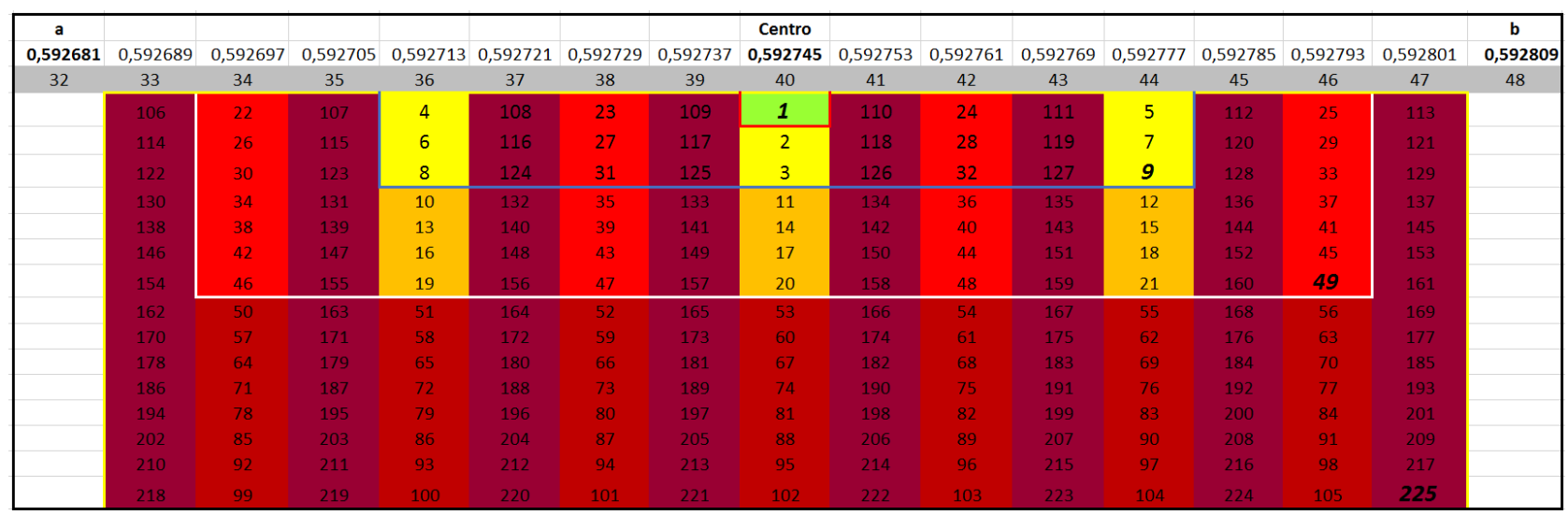

\section{RESULTADOS E DISCUSSÕES}

Mas para que usamos as sequências? No nosso algoritmo, usamos a sequência com repetição, onde $T=\left\{y_{n}\right\}$ é formada por elementos que serão as probabilidades informadas ( $\left.P_{\text {inf }}\right)$ ou limiares de percolação informados.

E como é feita a ocupação dos sítios da rede em uma execução? A rede quadrada de lado $L$ que trabalhamos é uma rede cartesiana de zero a $L$ por zero a $L$, que possui $L^{2}$ sítios vazios, onde o sítio $(1,1)$ é o sítio inferior esquerdo da rede. Consideramos os sítios externos horizontais $(1,0)$ a $(L, 0)$ como sendo sítios ocupados. Também consideramos os sítios externos verticais $(0,1)$ a $(0, L)$ como sendo sítios desocupados. Em cada execução, uma vez tendo o limiar de percolação informado, utilizamos o gerador de números aleatório para gerar números entre 0 e 1. Se o número gerado for menor ou igual ao limiar informado, o sítio da rede visitado é ocupado, caso contrário, o sítio é desocupado. Temos três situações para um sítio da rede: ocupado, desocupado e vazio (situação inicial, onde o sítio ainda não foi visitado). Escolhemos o sítio fora da rede $(1,0)$ para iniciar as visitas aos próximos sítios vazios. Este sítio será um centro de rotação no sentido horário e apontamos para o sítio $(1,1)$.

Caso o sítio $(1,1)$ seja ocupado, este será o novo centro de rotação no sentido horário e procuraremos, no sentido horário, o seu primeiro sítio vizinho vazio. A rotina recomeça apontando para o sítio anterior ocupado, neste caso, o sítio fora da rede $(1,0)$. Como este sítio está ocupado, giramos sentido horário e apontamos para o sítio $(0,1)$. Este sítio é um sítio desocupado fora da rede. Giramos no sentido horário e apontamos para o sítio $(1,2)$. Este é um sítio vazio, logo visitaremos e ocuparemos ou não, dependendo do valor do número aleatório gerado.

Caso o sítio $(1,1)$ não seja ocupado, apontamos para o sítio $(2,0)$, que é um sítio ocupado fora da rede. Este será o nosso próximo centro de rotação e a rotina recomeça apontando para o sítio anterior ocupado, neste caso, o sítio fora da rede $(1,0)$. Como este sítio está ocupado, giramos sentido horário e apontamos para o sítio $(2,1)$. Este é um sítio vazio, logo visitaremos e ocuparemos ou não, dependendo do valor do número aleatório gerado.

Como consideramos os sítios externos a rede $(1,0)$ a $(L, 0)$ como já sendo sítios ocupados, o aglomerado será percolante, se a fronteira formada por sítios visitados ocupados atingir o lado da rede formado por sítios de $(1, L)$ a $(L, L)$. Se a fronteira de sítios visitados ocupados atingir o lado da rede formado por sítios de $(L, 1)$ a $(L, L-1)$ o aglomerado não é percolante. 


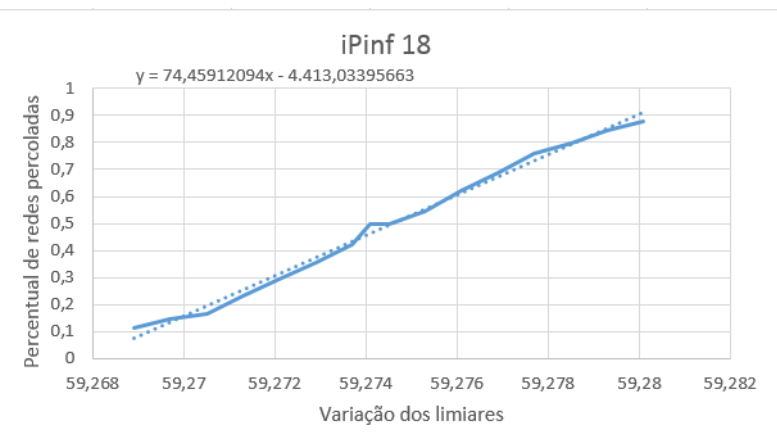

(a)

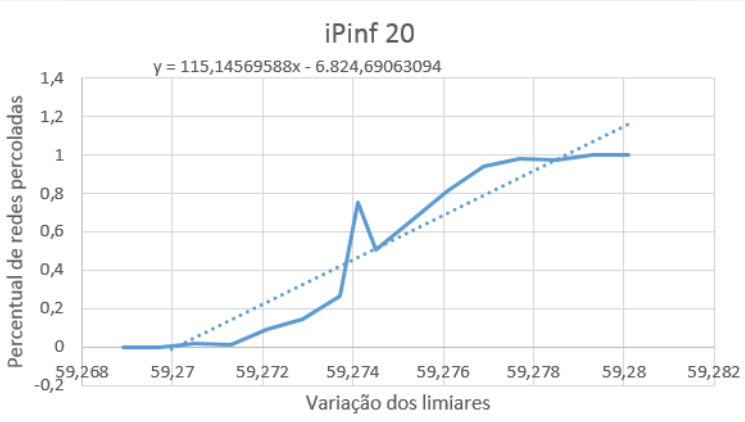

(c)

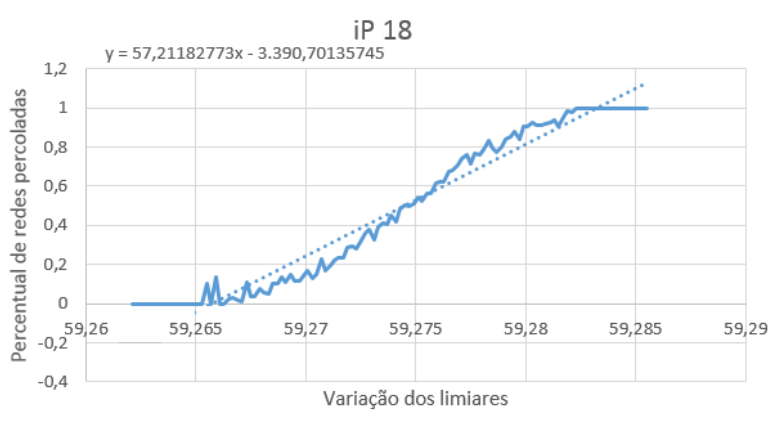

(e)

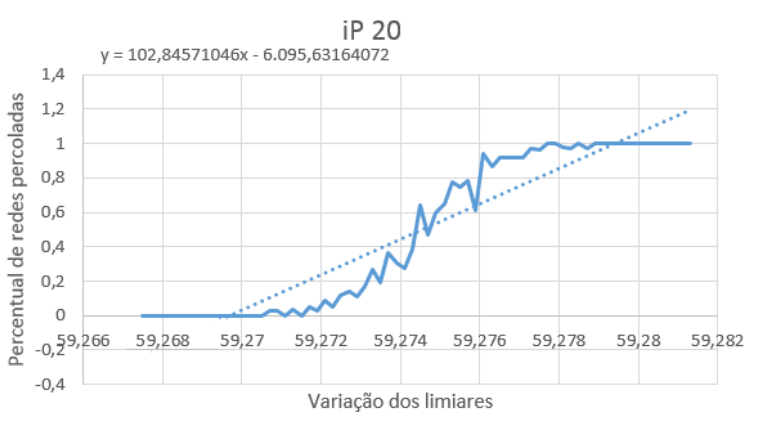

(g)

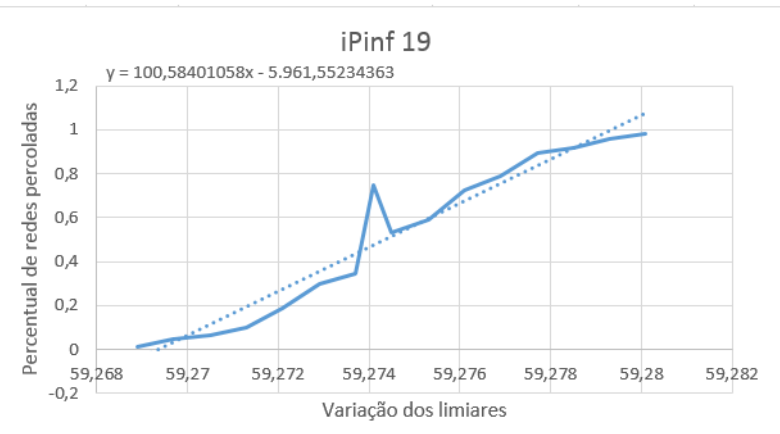

(b)

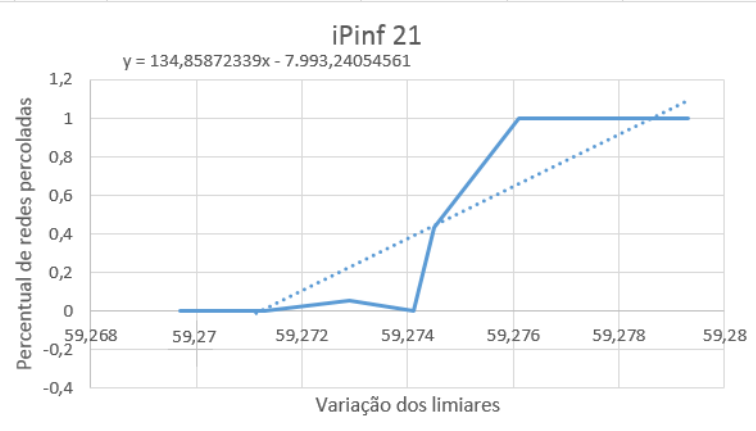

(d)

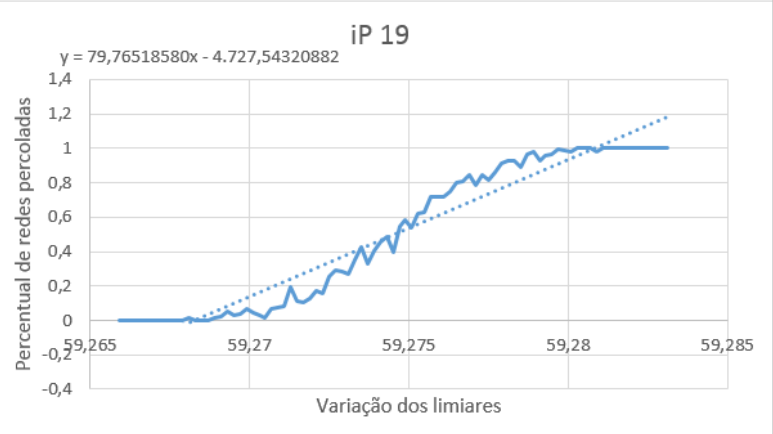

(f)

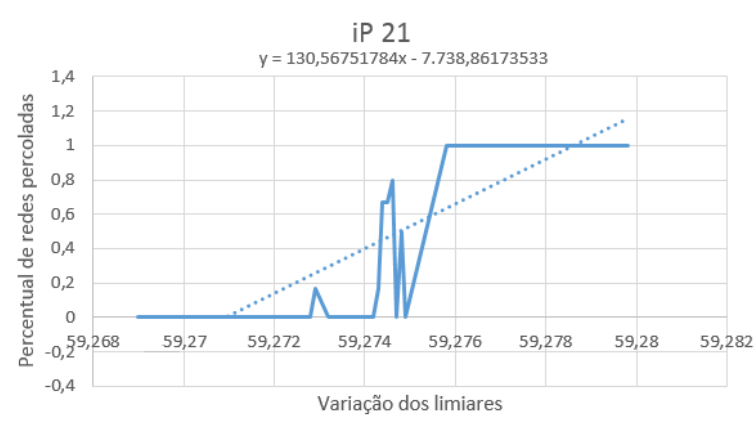

(h)

Figura 2: Limiar de percolação informado e limiar de percolação calculado para redes de lados $2^{18}, 2^{19}, 2^{20}$ e $2^{21}$ sítios.

Ao finalizar a execução de cada rede, que teve um limiar informado $\left(P_{\text {inf }}\right)$, geramos um arquivo com os dados referentes ao limiar de percolação. Os aglomerados são formados pela fronteira percolante. Executamos redes de tamanhos de lados $2^{11}$ a $2^{21}$ sítios e em cada rede usamos a sequência $y_{n}$ descrita anteriormente. Fizemos 8001 execuções para redes de lados $2^{11}$, $2^{12}$ e $2^{13} ; 3969$ execuções para redes de lados $2^{14}$ e $2^{15} ; 1953$ execuções para a rede de lado $2^{16}$; 
465 execuções para redes de lados $2^{17}, 2^{18}$ e $2^{19} ; 225$ execuções para a rede de lado $2^{20}$ e 105 execuções para a rede de lado $2^{21}$.

Ao ter uma quantidade de execuções concluídas, por exemplo 105, 225 ou 465, e de tamanho de lado $2^{r}$ sítios, com outro algoritmo criado por nós e chamado de Learq, lemos todos os arquivos gerados e fazemos as estatísticas de todos os tamanhos de lados de rede $2^{1}, 2^{2}, \ldots, 2^{r-1}, 2^{r}$ sítios, em um único arquivo organizado por limiar e por tamanho de rede. Com esse arquivo gerado, analisamos graficamente a distribuição de percolação por cada tamanho de rede e com suas faixas de limiar de percolação. Lembramos que ao executar uma rede de lado $2^{r}$ sítios, todas as redes de lados $2^{3}, 2^{4}, \ldots, 2^{r-1}$ sítios também têm suas estatísticas apuradas.

Com os dados separados por tamanho de lado da rede, fazemos as frequências de percolação para cada limiar. Em seguida usamos o recurso linha de tendência, de primeiro grau, para encontrarmos o limiar de percolação, o qual a quantidade de redes percoladas e não percoladas são de $50 \%$ (eixo das ordenadas).

Temos dois resultados a respeito de limiares de percolação: $P_{\text {inf }}$ (Limiar de percolação informado) e $\mathrm{P}$ (Limiar de percolação calculado). Analisamos nos dois casos a estatística de percolação, com as diversas redes geradas pelo algoritmo. Em redes de lado até $2^{15}$ sítios, que para os nossos resultados já consideramos redes "pequenas", é necessário um grande número de execuções, chegando a 8001 execuções, para que ocorra uma convergência para o limiar de percolação. Com os dados referentes ao limiar de percolação por rede, geramos uma reta aproximada pelo método de mínimos quadrados, onde no eixo das abscissas temos a variação dos limiares e no eixo das ordenadas o percentual de redes percoladas (frequência das execuções dos casos que percolam), a qual extraímos seus coeficientes angular e linear, por tamanho de rede, como visto na Figura 2.

Aumentando o lado da rede, o tempo de execução fica inviável para chegar a um número de execuções semelhante às redes menores. Dessa forma, com menos execuções, o ajuste linear para redes de lados $2^{20}$ e $2^{21}$ sítios fica menos preciso.

\section{CONCLUSÕES}

Com os coeficientes esboçamos três curvas, para nenhuma rede percolada (zero), $50 \%$ de redes percoladas e $100 \%$ de redes percoladas, onde variamos no eixo das abscissas $\frac{1}{2^{r}}$. Isso significa que próximo de zero, concentram-se as maiores redes. Vimos que as curvas onde nenhuma rede percola e as curvas em que todas as redes percolam são simétricas, além disso, próxima da abscissa zero, onde a rede tende ao infinito e 50 \% das redes percolam, a curva converge para o Limiar de percolação existente na literatura. 


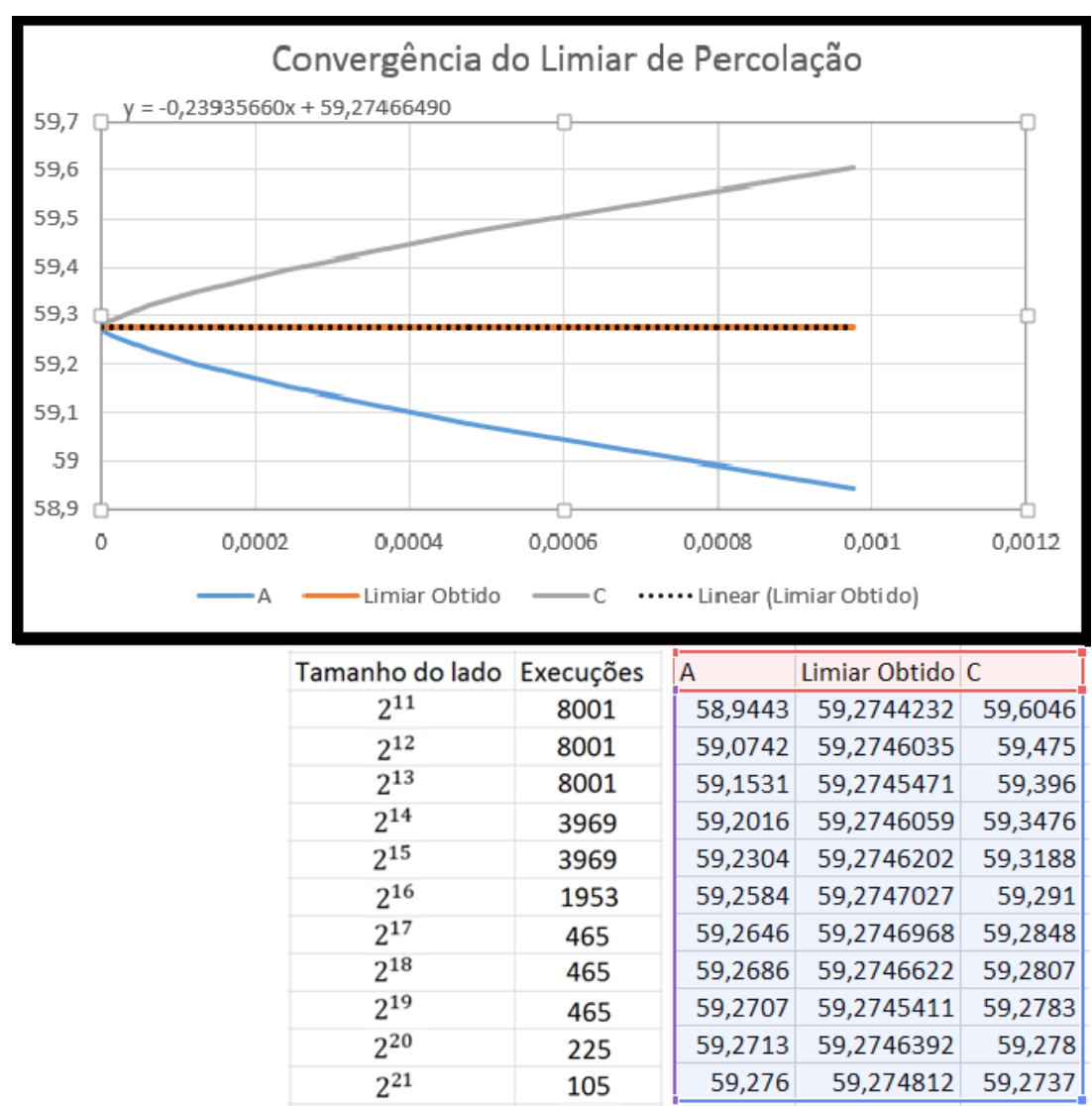

Figura 3: Convergência do limiar de percolação.

Em redes de lados maiores de que $2^{15}$ sítios, onde apuramos resultados estatísticos de redes de lado até $2^{21}$ sítios, a convergência do limiar de percolação ocorreu com um número menor de execuções. Na rede de lado $2^{21}$ sítios foram 105 vezes, mesmo que sejam execuções demoradas como na rede de lado $2^{23}$ sítios, precisando de mais de um dia cada execução (esta rede não foi usada nas estatísticas). Vale lembrar que não alcançamos lados maiores do que $2^{23}$ sítios, devido a limitação do espaço de disco dos nossos computadores (Hardware), podendo este tamanho ser ultrapassado.

Pelos estudos desenvolvidos, concluímos que o limiar de percolação se encontra num intervalo que vai se estreitando a medida que a rede se torna cada vez maior, confirmando as previsões feitas através de leis de escalas. Com as simulações realizadas chegamos aos seguintes limiares de percolação por tamanho de rede:

Tabela 3: Lado da rede, quantidade de execuções e limiar de percolação obtido.

\begin{tabular}{|c|c|r|}
\hline Tamanho do lado Execuções & Limiar Obtido \\
\cline { 3 - 3 } $2^{11}$ & 8001 & 59,2744232 \\
\hline $2^{12}$ & 8001 & 59,2746035 \\
\hline $2^{13}$ & 8001 & 59,2745471 \\
\hline $2^{14}$ & 3969 & 59,2746059 \\
\hline $2^{15}$ & 3969 & 59,2746202 \\
\hline $2^{16}$ & 1953 & 59,2747027 \\
\hline $2^{17}$ & 465 & 59,2746968 \\
\hline $2^{18}$ & 465 & 59,2746622 \\
\hline $2^{19}$ & 465 & 59,2745411 \\
\hline $2^{20}$ & 225 & 59,2746392 \\
\hline $2^{21}$ & 105 & 59,274812 \\
\hline
\end{tabular}


Trabalhos futuros na área de Matemática, Física, Estatística e Engenharia de Petróleo poderão ser feitos com este algoritmo que desenvolvemos. Por exemplo, com algumas adaptações e incrementos, já estamos iniciando o estudo da dimensão fractal de fronteira dos aglomerados percolantes e qual a ligação que possui com o limiar de percolação.

\section{REFERÊNCIAS}

1. STAUFFER, D., AHARONY, A., Introduction to Percolation Theory, second edition ed., Taylor and Francis, London, 1994.

2. STANLEY, H. E., Introduction to Phase Transition and Critical Phenomena, Oxford, 1971.

3. SAHIMI, M., Aplications of Percolation Theory, Taylor and Francis, London, 1994.

4. BROAdBenT, S. R., HAMMERSLEY, J. M., Percolation Processess I. Crystals and Mazes. Proceedings of the Cambridge Philosophical Society, 1957.

5. HUNT, A. G., Percolation Theory for Flow in Porous Media - Lecture Notes in Physics, Editora Springer, 2005.

6. GRIMMETT, G., Percolation, Springer-Verlang, 2nd Edition, Berlin Heidelberg, 1999.

7. SILVA, L. R., PAUL, G., HAVLIN, S., BAKER, D.R, STANLEY, H. E., Physica A, 314, 2003.

8. STANLEY, H. E., Introduction to Phase Transitions and Critical Phenomena. Oxfod University Press, Ney York, 1971.

9. WILKINSON, D., WILLEMSEN, J.F. Invasion percolation: a new form of percolation theory, J. Phys A, 1983.

10. FREITAS, J. E., LUCENA, L.S., ROUX, S., Physica A 266, 1999.

11. FREITAS, J. E., Estudos de Alguns Sistemas Complexos, Tese de Doutorado, UFRN. Natal-RN, Brasil, 2012. 